\title{
A refinement of the Kolmogorov-Marcinkiewicz-Zygmund strong law of large numbers
}

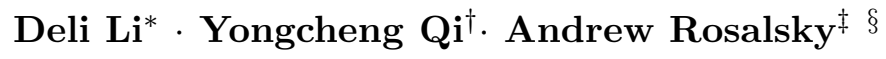

Abstract Let $\left\{X_{n} ; n \geq 1\right\}$ be a sequence of independent copies of a real-valued random variable $X$ and set $S_{n}=X_{1}+\cdots+X_{n}, n \geq 1$. This paper is devoted to a refinement of the classical Kolmogorov-Marcinkiewicz-Zygmund strong law of large numbers. We show that for $0<p<2$,

$$
\sum_{n=1}^{\infty} \frac{1}{n}\left(\frac{\left|S_{n}\right|}{n^{1 / p}}\right)<\infty \text { almost surely }
$$

if and only if

$$
\begin{cases}\mathbb{E}|X|^{p}<\infty, & \text { if } 0<p<1 \\ \mathbb{E} X=0, \sum_{n=1}^{\infty} \frac{|\mathbb{E} X I\{|X| \leq n\}|}{n}<\infty, \text { and } \sum_{n=1}^{\infty} \frac{\int_{\min \left\{u_{n}, n\right\}}^{n} \mathbb{P}(|X|>t) d t}{n}<\infty, & \text { if } p=1 \\ \mathbb{E} X=0 \text { and } \int_{0}^{\infty} \mathbb{P}^{1 / p}(|X|>t) d t<\infty, & \text { if } 1<p<2,\end{cases}
$$

where $u_{n}=\inf \left\{t: \mathbb{P}(|X|>t)<\frac{1}{n}\right\}, n \geq 1$. Versions of above results in a Banach space setting are also presented. To establish these results, we invoke the remarkable Hoffmann-Jørgensen (1974) inequality to obtain some general results for sums of the form $\sum_{n=1}^{\infty} a_{n}\left\|\sum_{i=1}^{n} V_{i}\right\|$ (where $\left\{V_{n} ; n \geq\right.$ $1\}$ is a sequence of independent Banach space valued random variables and $a_{n} \geq 0, n \geq 1$ ) which may be of independent interest but which we apply to $\sum_{n=1}^{\infty} \frac{1}{n}\left(\frac{\left|S_{n}\right|}{n^{1 / p}}\right)$.

Keywords Kolmogorov-Marcinkiewicz-Zygmund strong law of large numbers · Sums of i.i.d. random variables - Real separable Banach space - Rademacher type $p$ Banach space Stable type $p$ Banach space

Mathematics Subject Classification (2000) Primary: 60F15; Secondary: 60B12 - 60G50

Running Head: Strong law of large numbers

\section{Introduction and preliminaries}

Throughout, let $(\mathbf{B},\|\cdot\|)$ be a real separable Banach space equipped with its Borel $\sigma$-algebra $\mathcal{B}(=$ the $\sigma$-algebra generated by the class of open subsets of $\mathbf{B}$ determined by $\|\cdot\|)$ and let $\left\{X_{n} ; n \geq 1\right\}$ be a sequence of independent copies of a $\mathbf{B}$-valued random variable $X$ defined on a probability

\footnotetext{
${ }^{*}$ Deli Li, Department of Mathematical Sciences, Lakehead University, Thunder Bay, Ontario, Canada P7B 5E1 e-mail: dli@lakeheadu.ca

${ }^{\dagger}$ Department of Mathematics and Statistics, University of Minnesota Duluth, Duluth, Minnesota 55812, USA e-mail: yqi@d.umn.edu

${ }^{\ddagger}$ Andrew Rosalsky, Department of Statistics, University of Florida, Gainesville, Florida 32611, U.S.A. e-mail: rosalsky@stat.ufl.edu

${ }^{\S}$ Corresponding author: Andrew Rosalsky (Telephone: 1-352-273-2983, FAX: 1-352-392-5175)
} 
space $(\Omega, \mathcal{F}, \mathbb{P})$. As usual, let $S_{n}=\sum_{k=1}^{n} X_{k}, n \geq 1$ denote their partial sums. If $0<p<2$ and if $X$ is a real-valued random variable (that is, if $\mathbf{B}=\mathbb{R}$ ), then

$$
\lim _{n \rightarrow \infty} \frac{S_{n}}{n^{1 / p}}=0 \text { almost surely (a.s.) }
$$

if and only if

$$
\mathbb{E}|X|^{p}<\infty \text { where } \mathbb{E} X=0 \text { whenever } p \geq 1 \text {. }
$$

This is the celebrated Kolmogorov-Marcinkiewicz-Zygmund strong law of large numbers (SLLN); see Kolmogoroff [8] for $p=1$ and Marcinkiewicz and Zygmund [11] for $p \neq 1$.

The classical Kolmogorov SLLN in real separable Banach spaces was established by Mourier [14]. The extension of the Kolmogorov-Marcinkiewicz-Zygmund SLLN to B-valued random variables is independently due to Azlarov and Volodin [1] and de Acosta [3].

Theorem 1.1. (Azlarov and Volodin [1] and de Acosta [3]). Let $0<p<2$ and let $\left\{X_{n} ; n \geq 1\right\}$ be a sequence of independent copies of a $\mathbf{B}$-valued random variable $X$. Then

$$
\lim _{n \rightarrow \infty} \frac{S_{n}}{n^{1 / p}}=0 \quad \text { a.s. }
$$

if and only if

$$
\mathbb{E}\|X\|^{p}<\infty \text { and } \frac{S_{n}}{n^{1 / p}} \rightarrow \mathbb{P} 0
$$

De Acosta [3] also provides a remarkable characterization of Rademacher type $p$ Banach spaces. (Technical definitions such as $\mathbf{B}$ being of Rademacher type $p$ will be reviewed below.) Specifically, de Acosta [3] proved the following theorem.

Theorem 1.2. (de Acosta [3]). Let $1 \leq p<2$. Then the following two statements are equivalent:

(i) The Banach space $\mathbf{B}$ is of Rademacher type p.

(ii) For every sequence $\left\{X_{n} ; n \geq 1\right\}$ of independent copies of a $\mathbf{B}$-valued variable $X$,

$$
\lim _{n \rightarrow \infty} \frac{S_{n}}{n^{1 / p}}=0 \text { a.s. if and only if } \mathbb{E}\|X\|^{p}<\infty \text { and } \mathbb{E} X=0 \text {. }
$$

At the origin of the current investigation is the following recent and striking result by Hechner and Heinkel [4].

Theorem 1.3. (Hechner and Heinkel [4]). Suppose that $\mathbf{B}$ is of stable type $p(1<p<2)$ and let $\left\{X_{n} ; n \geq 1\right\}$ be a sequence of independent copies of a $\mathbf{B}$-valued variable $X$ with $\mathbb{E} X=0$. Then

$$
\sum_{n=1}^{\infty} \frac{1}{n}\left(\frac{\mathbb{E}\left\|S_{n}\right\|}{n^{1 / p}}\right)<\infty
$$

if and only if

$$
\int_{0}^{\infty} \mathbb{P}^{1 / p}(\|X\|>t) d t<\infty
$$


This result of Hechner and Heinkel [4] is new even in the case where the Banach space $\mathbf{B}$ is the real line. We note that, under the hypotheses of Theorem 1.3, (1.1) and (1.2) each imply that

$$
\lim _{n \rightarrow \infty} \frac{\mathbb{E}\left\|S_{n}\right\|}{n^{1 / p}}=0
$$

This follows from Theorem 1.3, Remark 2.2 below, Theorem 1.2, the fact that $\mathbf{B}$ is of Rademacher type $p$ (see the discussion below), and Theorem 2 of Korzeniowski [9]. This result of Korzeniowski extends to a Banach space setting Corollary 12 of Klass [7].

Inspired by the above discovery by Hechner and Heinkel [4], in the current work we obtain sets of necessary and sufficient conditions for

$$
\sum_{n=1}^{\infty} \frac{1}{n}\left(\frac{\left\|S_{n}\right\|}{n^{1 / p}}\right)<\infty \text { a.s. }
$$

for the three cases: $0<p<1, p=1,1<p<2$. Moreover, we obtain necessary and sufficient conditions for

$$
\sum_{n=1}^{\infty} \frac{1}{n}\left(\frac{\mathbb{E}\left\|S_{n}\right\|}{n}\right)<\infty
$$

Again, these results are new when $\mathbf{B}=\mathbb{R}$; see Theorem 2.5. The current work complements the investigation by Hechner and Heinkel [4].

While it is immediate that (1.1) implies (1.3), we will see that (1.1) and (1.3) are indeed equivalent if $1<p<2$ (Theorem 2.1). However, there is a gap between the cases $0<p \leq 1$ and $1<p<2$ as we will see that if $0<p \leq 1$, then (1.4) and (1.3) are not equivalent (Theorems 2.2 and 2.4). Moreover, Theorems 2.2 and 2.4 also reveal that there is a gap between the cases $p=1$ and $0<p<1$.

The most delicate case is that for $p=1$. We show assuming $\mathbf{B}$ is of stable type 1 that (1.3) holds if and only if the conditions (2.11), (2.12), and (2.13) are fulfilled (Theorem 2.3).

We now review various technical definitions pertaining to a $\mathbf{B}$-valued random variable $X$ or to the Banach space $\mathbf{B}$ itself.

The expected value or mean of $X$, denoted $\mathbb{E} X$, is defined to be the Pettis integral provided it exists. That is, $X$ has expected value $\mathbb{E} X \in \mathbf{B}$ if $\varphi(\mathbb{E} X)=\mathbb{E} \varphi(X)$ for every $\varphi \in \mathbf{B}^{*}$ where $\mathbf{B}^{*}$ denotes the (dual) space of all continuous linear functionals on $\mathbf{B}$. If $\mathbb{E}\|X\|<\infty$, then (see, e.g., Taylor $[17$, p. 40]) $X$ has an expected value. But the expected value can exist when $\mathbb{E}\|X\|=\infty$. For an example, see Taylor [17, p. 41].

Let $\left\{R_{n} ; n \geq 1\right\}$ be a Rademacher sequence; that is, $\left\{R_{n} ; n \geq 1\right\}$ is a sequence of independent and identically distributed (i.i.d.) random variables with $\mathbb{P}\left(R_{1}=1\right)=\mathbb{P}\left(R_{1}=-1\right)=1 / 2$. Let $\mathbf{B}^{\infty}=\mathbf{B} \times \mathbf{B} \times \mathbf{B} \times \cdots$ and define

$$
\mathcal{C}(\mathbf{B})=\left\{\left(v_{1}, v_{2}, \ldots\right) \in \mathbf{B}^{\infty}: \sum_{n=1}^{\infty} R_{n} v_{n} \text { converges in probability }\right\} .
$$

Let $1 \leq p \leq 2$. Then $\mathbf{B}$ is said to be of Rademacher type $p$ if there exists a constant $0<C<\infty$ such that

$$
\mathbb{E}\left\|\sum_{n=1}^{\infty} R_{n} v_{n}\right\|^{p} \leq C \sum_{n=1}^{\infty}\left\|v_{n}\right\|^{p} \text { for all }\left(v_{1}, v_{2}, \ldots\right) \in \mathcal{C}(\mathbf{B}) .
$$

Hoffmann-Jørgensen and Pisier [6] proved for $1 \leq p \leq 2$ that $\mathbf{B}$ is of Rademacher type $p$ if and only if there exists a constant $0<C<\infty$ such that

$$
\mathbb{E}\left\|\sum_{k=1}^{n} V_{k}\right\|^{p} \leq C \sum_{k=1}^{n} \mathbb{E}\left\|V_{k}\right\|^{p}
$$


for every finite collection $\left\{V_{1}, \ldots, V_{n}\right\}$ of independent mean 0 B-valued random variables.

If $\mathbf{B}$ is of Rademacher type $p$ for some $p \in(1,2]$, then it is of Rademacher type $q$ for all $q \in[1, p)$. Every real separable Banach spaces is of Rademacher type (at least) 1.

Let $0<p \leq 2$ and let $\left\{\Theta_{n} ; n \geq 1\right\}$ be a sequence of i.i.d. stable random variables each with characteristic function $\psi(t)=\exp \left\{-|t|^{p}\right\}, \quad-\infty<t<\infty$. Then $\mathbf{B}$ is said to be of stable type $p$ if $\sum_{n=1}^{\infty} \Theta_{n} v_{n}$ converges a.s. whenever $\left\{v_{n}: n \geq 1\right\} \subseteq \mathbf{B}$ with $\sum_{n=1}^{\infty}\left\|v_{n}\right\|^{p}<\infty$. Equivalent characterizations of a Banach space being of stable type $p$, properties of stable type $p$ Banach spaces, as well as various relationships between the conditions "Rademacher type $p$ " and "stable type $p$ " may be found in Maurey and Pisier [12], Woyczyński [18], Marcus and Woyczyński [13], Rosiński [16], Pisier [15], and Ledoux and Talagrand [10]. Some of these properties and relationships will now be summarized:

(1) Every real separable Banach space $\mathbf{B}$ is of stable type $p$ for all $p \in(0,1)$.

(2) For $1 \leq p<2$, $\mathbf{B}$ is of stable type $p$ if and only if $\mathbf{B}$ is of Rademacher type $p_{1}$ for some $p_{1} \in(p, 2]$.

(3) $\mathbf{B}$ is of stable type 2 if and only if $\mathbf{B}$ is of Rademacher type 2 .

Consequently:

(4) If $\mathbf{B}$ is of stable type $p$ for some $p \in[1,2]$, then $\mathbf{B}$ is of Rademacher type $p$.

(5) If $\mathbf{B}$ is of stable type $p$ for some $p \in[1,2]$, then $\mathbf{B}$ is of stable type $q$ for all $q \in(0, p)$.

(6) If $\mathbf{B}$ is of stable type $p$ for some $p \in[1,2)$, then $\mathbf{B}$ is of stable type $q$ for some $q \in(p, 2]$.

The property (6) is the Maurey-Pisier [12] theorem.

For $q \geq 2$, the $\mathcal{L}_{q}$-spaces and $\ell_{q}$-spaces are of stable type 2 while for $1 \leq q<2$, the $\mathcal{L}_{q}$-spaces and $\ell_{q}$-spaces are of Rademacher type $q$ and are of stable type $p$ for all $p \in(0, q)$ but are not of stable type $q$. Every real separable Hilbert space and real separable finite dimensional Banach space is of stable type 2 . In particular, the real line is of stable type 2 .

The plan of the paper is as follows. The main results are stated in Section 2. Some general results for $\sum_{n=1}^{\infty} a_{n}\left\|\sum_{k=1}^{n} V_{k}\right\|$ (where the $a_{n} \geq 0$ and $\left\{V_{k} ; k \geq 1\right\}$ is a sequence of independent $\mathbf{B}$-valued random variables) is established in Section 3; these results are key components in the proofs of the main results. The main results are proved in Sections 4 and 5 .

Finally, the symbol $C$ denotes throughout a generic constant $(0<C<\infty)$ which is not necessarily the same one in each appearance.

\section{Statement of the main results}

With the preliminaries accounted for, the main results may be stated. We begin with the case where $1<p<2$.

Theorem 2.1. Let $\left\{X_{n} ; n \geq 1\right\}$ be a sequence of independent copies of a $\mathbf{B}$-valued random variable $X$. Let $1<p<2$. Then

$$
\sum_{n=1}^{\infty} \frac{1}{n}\left(\frac{\mathbb{E}\left\|S_{n}\right\|}{n^{1 / p}}\right)<\infty
$$


if and only if

$$
\sum_{n=1}^{\infty} \frac{1}{n}\left(\frac{\left\|S_{n}\right\|}{n^{1 / p}}\right)<\infty \text { a.s. }
$$

Furthermore, each of (2.1) and (2.2) implies that

$$
\mathbb{E} X=0 \text { and } \int_{0}^{\infty} \mathbb{P}^{1 / p}(\|X\|>t) d t<\infty
$$

and

$$
\lim _{n \rightarrow \infty} \frac{S_{n}}{n^{1 / p}}=0 \quad \text { a.s. }
$$

Remark 2.1. The conditions (2.3) and (2.4) do not necessarily imply that (2.1) or (2.2) hold. A counterexample provided by Hechner and Heinkel [4, Section 5] is such that (2.3) and (2.4) hold but (2.1) fails. Since (2.1) and (2.2) are equivalent, (2.2) also fails for that counterexample. The counterexample pertained to the Banach space $\ell_{p}(1<p<2)$.

Remark 2.2. For $1<p<2$, the second half of (2.3) implies that $\mathbb{E}\|X\|^{p}<\infty$ as was noted by Hechner and Heinkel [4]. But the converse implication is false. To see this, suppose that

$$
\mathbb{P}(\|X\|>t)=\frac{e^{e p+1}}{t^{p}(\ln t)(\ln \ln t)^{\alpha}}, t \geq e^{e}
$$

where $\alpha>1$. Then $\mathbb{E}\|X\|^{p}<\infty$ but

$$
\int_{0}^{\infty} \mathbb{P}^{1 / p}(\|X\|>t) d t=C+\int_{e^{e}}^{\infty} \frac{e^{e+p^{-1}}}{t(\ln t)^{1 / p}(\ln \ln t)^{\alpha / p}} d t=\infty .
$$

Combining Theorem 2.1 above and Theorem 5 of Hechner and Heinkel [4], we immediately obtain the following result.

Corollary 2.1. Let $X$ be a $\mathbf{B}$-valued random variable and let $1<p<2$. If $\mathbf{B}$ is of stable type $p$, then (2.1), (2.2), and (2.3) are equivalent.

Remark 2.3. The example of Hechner and Heinkel [4, Section 5] referred to in Remark 2.1 above also shows that in Corollary 2.1, the stable type $p(1<p<2)$ hypothesis cannot be weakened to a Rademacher type $p(1<p<2)$ hypothesis.

Remark 2.4. An example of Hechner and Heinkel [4, Example 1] shows that Corollary 2.1 can fail if the condition (2.3) is weakened to

$$
\mathbb{E} X=0 \text { and } \mathbb{E}\|X\|^{p}<\infty
$$

We now consider the case where $p=1$. In general (2.1) and (2.2) are not equivalent when $p=1$; see Remark 2.7 below. But we have the following result. 
Theorem 2.2. Let $X$ be a $\mathbf{B}$-valued random variable $X$. Then

$$
\sum_{n=1}^{\infty} \frac{1}{n}\left(\frac{\mathbb{E}\left\|S_{n}\right\|}{n}\right)<\infty
$$

if and only if

$$
\sum_{n=1}^{\infty} \frac{1}{n}\left(\frac{\left\|S_{n}\right\|}{n}\right)<\infty \quad \text { a.s. }
$$

and

$$
\mathbb{E} X=0 \text { and } \mathbb{E}\|X\| \ln (1+\|X\|)<\infty .
$$

In the next theorem, we provide necessary and sufficient conditions for (2.6) to hold assuming that $\mathbf{B}$ is of stable type 1 . It is the most delicate result in this paper.

Let $X$ be a $\mathbf{B}$-valued random variable. For each $n \geq 1$, we define the quantile $u_{n}$ of order $\left(1-\frac{1}{n}\right)$ of $\|X\|$ as follows:

$$
u_{n}=\inf \left\{t: \mathbb{P}(\|X\| \leq t)>1-\frac{1}{n}\right\}=\inf \left\{t: \mathbb{P}(\|X\|>t)<\frac{1}{n}\right\} .
$$

If

$$
\mathbb{E}\|X\|<\infty
$$

then it is easy to show that

$$
\lim _{n \rightarrow \infty} \frac{u_{n}}{n}=0
$$

Theorem 2.3. Let $\mathbf{B}$ be a Banach space of stable type 1 . Let $\left\{X_{n} ; n \geq 1\right\}$ be a sequence of independent copies of a $\mathbf{B}$-valued random variable $X$. Then

$$
\sum_{n=1}^{\infty} \frac{1}{n}\left(\frac{\left\|S_{n}\right\|}{n}\right)<\infty \text { a.s. }
$$

if and only if following three conditions are fulfilled:

$$
\begin{gathered}
\mathbb{E}\|X\|<\infty \text { and } \mathbb{E} X=0 ; \\
\sum_{n=1}^{\infty} \frac{\|\mathbb{E} X I\{\|X\| \leq n\}\|}{n}<\infty ; \\
\sum_{n=1}^{\infty} \frac{\int_{\min \left\{u_{n}, n\right\}}^{n} \mathbb{P}(\|X\|>t) d t}{n}<\infty .
\end{gathered}
$$

Remark 2.5. Set

$$
G(t)=\mathbb{P}(\|X\|>t), \quad \ell(t)=\sum_{t \leq n<1 / G(t)} \frac{1}{n}, \quad \text { and } \pi(t)=\sum_{n=1}^{\infty} \frac{1}{n} I_{\left(u_{n}, n\right]}(t), \quad t \geq 0 .
$$

Since

$$
u_{n}<t \leq n \Longrightarrow t \leq n<\frac{1}{G(t)} \Longrightarrow u_{n} \leq t \leq n
$$


we have $\pi(t) \leq \ell(t)$ for all $t \geq 0$ with equality holding if $t \neq u_{n}$ for all $n \geq 1$. Hence we have

$$
\int_{0}^{\infty} \pi(t) G(t) d t=\sum_{n=1}^{\infty} \frac{1}{n} \int_{\min \left\{u_{n}, n\right\}}^{n} G(t) d t=\int_{0}^{\infty} \ell(t) G(t) d t
$$

and so we see that (2.13) is equivalent to

$$
\int_{0}^{\infty} \ell(t) G(t) d t<\infty
$$

An elementary computation shows that

$$
\ell(t)=\ln ^{+} \frac{1}{Q(t)}+\gamma(t) \text { where } Q(t)=(t+1) G(t) \quad \text { and }|\gamma(t)| \leq \frac{1}{t+1} \text { for all } t \geq 0 .
$$

Hence, if $\mathbb{E}\|X\|<\infty$, we see that (2.13) is equivalent to

$$
\int_{0}^{\infty} G(t) \ln ^{+} \frac{1}{Q(t)} d t<\infty
$$

For instance, if $G(t) \leq C(t \ln t)^{-1}(\ln \ln t)^{-\beta}$ for $t \geq 16$ where $\beta>2$, then (2.14) and (2.13) hold but we may have $\mathbb{E}\|X\| \ln ^{\delta}(1+\|X\|)=\infty$ for all $\delta>0$.

Combining Theorems 2.2 and 2.3, we obtain the following result.

Corollary 2.2. Let $X$ be a $\mathbf{B}$-valued random variable. If $\mathbf{B}$ is of stable type 1, then (2.5) and (2.7) are equivalent.

Another corollary of Theorem 2.3 is as follows.

Corollary 2.3. Suppose $\mathbf{B}$ is of stable type 1 and let $X$ be a symmetric $\mathbf{B}$-valued random variable. Then (2.6) holds if

$$
\mathbb{E}\|X\| \ln ^{\delta}(1+\|X\|)<\infty \text { for some } \delta>0 \text {. }
$$

Remark 2.6. It follows from Corollary 2.3 that the moment condition (2.7) in the implication $((2.7) \Rightarrow(2.6))$ which is immediate from Corollary 2.2 can be weakened to (2.15) if it is assumed that $X$ is symmetric.

Remark 2.7. When $p=1$, (2.1) and (2.2) are not equivalent. To see this, let $\left\{X_{n} ; n \geq 1\right\}$ be a sequence of independent copies of a symmetric real-valued random variable $X$ with

$$
\mathbb{E}|X| \ln (1+|X|)=\infty \text { but } \mathbb{E}|X| \ln ^{\delta}(1+|X|)<\infty \text { for some } 0<\delta<1 .
$$

Then by Corollary 2.3, (2.2) holds with $p=1$ but by Corollary 2.2 (or by Theorem 2.2), (2.1) fails with $p=1$.

We now consider the case where $0<p<1$. 
Theorem 2.4. Let $X$ be a B-valued random variable and let $0<p<1$. Then

$$
\sum_{n=1}^{\infty} \frac{1}{n}\left(\frac{\left\|S_{n}\right\|}{n^{1 / p}}\right)<\infty \quad \text { a.s. }
$$

if and only if

$$
\mathbb{E}\|X\|^{p}<\infty
$$

Furthermore,

$$
\sum_{n=1}^{\infty} \frac{1}{n}\left(\frac{\mathbb{E}\left\|S_{n}\right\|}{n^{1 / p}}\right)<\infty
$$

if and only if

$$
\mathbb{E}\|X\|<\infty
$$

The proofs of Theorems 2.1, 2.2, and 2.4 will be given in Section 4. Theorem 2.3 and Corollaries 2.2 and 2.3 will be proved in Section 5. For illustrating the conditions (2.11), (2.12), and (2.13) of Theorem 2.3, Section 5 also contains some examples.

We now summarize our Theorems 2.1-2.4 and Corollaries 2.1-2.3 for a real-valued random variable $X$. For $1<p<2$, the equivalence of (iii) and (iv) has recently been discovered by Hechner and Heinkel [4] (see Theorem 1.3 above) assuming $\mathbb{E} X=0$ for the implication ((iii) $\Rightarrow$ (iv)).

Theorem 2.5. Let $\left\{X_{n} ; n \geq 1\right\}$ be a sequence of independent copies of a real-valued random variable $X$. For $0<p<2$, the following two statements are equivalent:

(i) $\sum_{n=1}^{\infty} \frac{1}{n}\left(\frac{\left|S_{n}\right|}{n^{1 / p}}\right)<\infty$ a.s.,

(ii) $\begin{cases}\mathbb{E}|X|^{p}<\infty, & \text { if } 0<p<1 \\ \mathbb{E} X=0, \quad \sum_{n=1}^{\infty} \frac{|\mathbb{E} X I\{|X| \leq n\}|}{n}<\infty, & \text { if } p=1 \\ \text { and } \sum_{n=1}^{\infty} \frac{\int_{\min \left\{u_{n}, n\right\}}^{n} \mathbb{P}(|X|>t) d t}{n}<\infty, & \text { if } 1<p<2 . \\ \mathbb{E} X=0 \text { and } \int_{0}^{\infty} \mathbb{P}^{1 / p}(|X|>t) d t<\infty, & \text { if } 1<p \text { ar }\end{cases}$

For $0<p<2$, the following two statements are equivalent:

(iii) $\sum_{n=1}^{\infty} \frac{1}{n}\left(\frac{\mathbb{E}\left|S_{n}\right|}{n^{1 / p}}\right)<\infty$,

(iv) $\begin{cases}\mathbb{E}|X|<\infty, & \text { if } 0<p<1 \\ \mathbb{E} X=0 \text { and } \mathbb{E}|X| \ln (1+|X|)<\infty, & \text { if } p=1 \\ \mathbb{E} X=0 \text { and } \int_{0}^{\infty} \mathbb{P}^{1 / p}(|X|>t) d t<\infty, & \text { if } 1<p<2 .\end{cases}$

Furthermore, for $1<p<2$, the three statements (i), (ii), and (iii) are equivalent and anyone of them implies

$$
\lim _{n \rightarrow \infty} \frac{S_{n}}{n^{1 / p}}=0 \quad \text { a.s. }
$$


Additionally, for the case $p=1$, if

$$
X \text { is symmetric with } \mathbb{E}|X| \ln ^{\delta}(1+|X|)<\infty \text { for some } \delta>0
$$

or

$$
\mathbb{E} X=0 \text { and } \mathbb{E}|X| \ln (1+|X|)<\infty,
$$

then

$$
\sum_{n=1}^{\infty} \frac{1}{n}\left(\frac{\left|S_{n}\right|}{n}\right)<\infty \text { a.s. }
$$

\section{Some general results for $\sum_{n=1}^{\infty} a_{n}\left\|\sum_{k=1}^{n} V_{k}\right\|$}

In this section, by using the remarkable Hoffmann-Jørgensen [5] inequality, we establish in Theorem 3.1 some general results for sums of the form $\sum_{n=1}^{\infty} a_{n}\left\|\sum_{k=1}^{n} V_{k}\right\|\left(a_{n} \in[0, \infty)\right)$. These results will be used for proving the main results and they may be of independent interest. Theorem 3.1 is a modified version of the authors' original result and this modification and its elegant proof were so kindly presented to us by the Referee.

The following lemma will be used in the current section and in Sections 4 and 5 .

Lemma 3.1. Let $g:[0, \infty) \rightarrow[0, \infty)$ be a convex function and let $\left\{Y_{1}, \ldots, Y_{n}\right\}$ be a set of $n \geq 2$ independent $\mathbf{B}$-valued random variables such that $\mathbb{E} g\left(\left\|Y_{i}\right\|\right)<\infty$ for $1 \leq i \leq n$. Then we have:

(i) If $\mathbb{E}\left\|Y_{2}\right\|<\infty$, then

$$
\mathbb{E} g\left(\left\|Y_{1}-\mathbb{E} Y_{2}\right\|\right) \leq \mathbb{E} g\left(\left\|Y_{1}-Y_{2}\right\|\right)
$$

Hence, in particular,

$$
\mathbb{E}\left\|Y_{1}-\mathbb{E} Y_{2}\right\| \leq \mathbb{E}\left\|Y_{1}-Y_{2}\right\| .
$$

(ii) If $\mathbb{E} Y_{1}=\cdots=\mathbb{E} Y_{n}=0$, then

$$
\mathbb{E} g\left(\max _{1 \leq k \leq n}\left\|Y_{k}\right\|\right) \leq 2 \mathbb{E} g\left(2\left\|\sum_{k=1}^{n} Y_{k}\right\|\right) .
$$

Hence, in particular,

$$
\mathbb{E}\left(\max _{1 \leq k \leq n}\left\|Y_{k}\right\|\right) \leq 4 \mathbb{E}\left\|\sum_{k=1}^{n} Y_{k}\right\|
$$

Proof Applying (2.5) of Ledoux and Talagrand [10, p. 46], part (i) follows immediately. Let $\left\{R_{1}, \ldots, R_{n}\right\}$ be independent Rademacher random variables independent of $\left\{Y_{1}, \ldots, Y_{n}\right\}$. Since $\mathbb{E} Y_{1}=$ $\cdots=\mathbb{E} Y_{n}=0$, by Proposition 2.3 of Ledoux and Talagrand [10, p. 47] and Lemma 6.3 of Ledoux and Talagrand [10, p. 152], we have

$$
\mathbb{E} g\left(\max _{1 \leq k \leq n}\left\|Y_{k}\right\|\right)=\mathbb{E} g\left(\max _{1 \leq k \leq n}\left\|R_{k} Y_{k}\right\|\right) \leq 2 \mathbb{E} g\left(\left\|\sum_{k=1}^{n} R_{k} Y_{k}\right\|\right) \leq 2 \mathbb{E} g\left(2\left\|\sum_{k=1}^{n} Y_{k}\right\|\right)
$$

proving part (ii). 
Theorem 3.1. Let $\left\{V_{n} ; n \geq 1\right\}$ be a sequence of independent $\mathbf{B}$-valued random variables and let $\left\{a_{n} ; n \geq 1\right\}$ be a sequence of nonnegative numbers such that $\sum_{n=1}^{\infty} a_{n}<\infty$. Set

$$
\begin{gathered}
b_{n}=\sum_{i=n}^{\infty} a_{i}, \quad T_{n}=\sum_{i=1}^{n} V_{i}, \quad n \geq 1, \quad N=\sup _{n \geq 1} b_{n}\left\|V_{n}\right\|, \quad L=\sum_{n=1}^{\infty} a_{n}\left\|T_{n}\right\|, \\
M=\sum_{n=1}^{\infty} b_{n}\left\|V_{n}\right\|, \quad \Gamma(t)=\sum_{n=1}^{\infty} \mathbb{P}\left(b_{n}\left\|V_{n}\right\|>t\right), \quad t \geq 0 .
\end{gathered}
$$

Then we have $L \leq M$. Suppose that $\sup _{n \geq 1} \mathbb{P}\left(b_{n}\left\|V_{n}\right\|>a\right)<1$ for some $a \geq 0$. Then we have the following five conclusions:

(a) $L<\infty$ a.s. $\Longrightarrow N<\infty$ a.s. $\Longleftrightarrow \inf _{r \geq 0} \Gamma(r)<\infty$.

(b) $\mathbb{E} N<\infty \Longleftrightarrow \int_{r}^{\infty} \Gamma(t) d t<\infty$ for some $r \geq 0$.

(c) $\mathbb{E} L<\infty \Longleftrightarrow L<\infty$ a.s. and $\mathbb{E} N<\infty$.

(d) $\mathbb{E} L<\infty \Longrightarrow b_{n} \mathbb{E}\left\|V_{n}\right\|<\infty \forall n \geq 1$ and $\lim _{n \rightarrow \infty} b_{n} \mathbb{E}\left\|T_{n}-\mathbb{E} T_{n}\right\|=0$.

(e) $\sum_{n=1}^{\infty} b_{n}=\infty$ and $L<\infty$ a.s. $\Longrightarrow \liminf _{n \rightarrow \infty} \frac{\left\|T_{n}\right\|}{n}=0$ a.s.

Proof Clearly we have

$$
L \leq \sum_{n=1}^{\infty} a_{n} \sum_{i=1}^{n}\left\|V_{i}\right\|=\sum_{i=1}^{\infty}\left(\sum_{i=n}^{\infty} a_{i}\right)\left\|V_{i}\right\|=\sum_{i=1}^{\infty} b_{i}\left\|V_{i}\right\|=M
$$

By the Kolmogorov 0-1 law, we have $\mathbb{P}(N=\infty)=0$ or 1 and since $1-x \leq e^{-x}$, we have that, for all $t \geq 0$

$$
1-e^{-\Gamma(t)} \leq 1-\prod_{n=1}^{\infty} \mathbb{P}\left(b_{n}\left\|V_{n}\right\| \leq t\right)=\mathbb{P}(N>t) \leq \Gamma(t) .
$$

Hence, we see that the equivalence in (a) holds. We will verify below that $L<\infty$ a.s. $\Longrightarrow N<\infty$ a.s.

Suppose that $\Gamma(r)<\infty$. Since $\Gamma(t)$ and $h(x)=\left(1-e^{-x}\right) / x$ are decreasing (with $\left.h(0):=1\right)$, we have $\gamma \Gamma(t) \leq 1-e^{-\Gamma(t)}$ for all $t \geq r$ where $\gamma=h(\Gamma(r))$ and since $\gamma>0$, we see that (b) holds.

Let $\left\{V_{n}^{\prime} ; n \geq 1\right\}$ be an independent copy of $\left\{V_{n} ; n \geq 1\right\}$ and let $\hat{V}_{n}=V_{n}-V_{n}^{\prime}, n \geq 1$. Let $\hat{T}_{n}, \hat{L}, \hat{N}$ and $\hat{\Gamma}(t)$ be defined as above with $\left\{V_{n} ; n \geq 1\right\}$ replaced by $\left\{\hat{V}_{n} ; n \geq 1\right\}$. By assumption there exist $\lambda>0, a>0$ such that $\mathbb{P}\left(b_{n}\left\|V_{n}\right\| \leq a\right) \geq \lambda$ for all $n \geq 1$. Hence, by the symmetrization inequality we have $\lambda \Gamma(t+a) \leq \hat{\Gamma}(t)$ for all $t \geq 0$; see (6.1) in [10, p. 150]. Let $\|x\|_{1}:=\sum_{i=1}^{n}\left\|x_{i}\right\|<\infty$ denote the $\ell^{1}$-norm on $\mathbf{B}^{n}$. Then $\left(\mathbf{B}^{n},\|\cdot\|_{1}\right)$ is a real separable Banach space and if we set

$$
Y_{i}^{(n)}=\left(0, \ldots, 0, a_{i} \hat{V}_{i}, \ldots, a_{n} \hat{V}_{i}\right), \quad i=1, \ldots, n,
$$

then $Y_{1}^{(n)}, \ldots, Y_{n}^{(n)}$ are independent symmetric $\mathbf{B}^{n}$-valued random variables satisfying

$$
\left\|Y_{i}^{(n)}\right\|_{1}=\left(\sum_{k=i}^{n} a_{k}\right)\left\|\hat{V}_{i}\right\|=\left(b_{i}-b_{n+1}\right)\left\|\hat{V}_{i}\right\|, \quad i=1, \ldots, n, \quad\left\|\sum_{i=1}^{n} Y_{i}^{(n)}\right\|_{1}=\sum_{i=1}^{n} a_{i}\left\|\hat{T}_{i}\right\| .
$$


Hence, by Proposition 2.3 in [10, p. 47] and (3.3) in [6] we have that, for all $t \geq 0$,

$$
\mathbb{P}\left(\hat{N}_{n}>t\right) \leq 2 \mathbb{P}\left(\hat{L}_{n}>t\right), \mathbb{P}\left(\hat{L}_{n}>3 t\right) \leq \mathbb{P}\left(\hat{N}_{n}>t\right)+4 \mathbb{P}^{2}\left(\hat{L}_{n}>t\right),
$$

where

$$
\hat{N}_{n}=\max _{1 \leq i \leq n}\left\|Y_{i}^{(n)}\right\|_{1}=\max _{1 \leq i \leq n}\left(b_{i}-b_{n+1}\right)\left\|\hat{V}_{i}\right\| \text { and } \hat{L}_{n}=\left\|\sum_{i=1}^{n} Y_{i}^{(n)}\right\|_{1}=\sum_{i=1}^{n} a_{i}\left\|\hat{T}_{i}\right\| .
$$

Since $\hat{N}_{n} \nearrow \hat{N}$ and $\hat{L}_{n} \nearrow \hat{L}$ as $n \rightarrow \infty$, we have that, for all $t \geq 0$,

$$
\mathbb{P}(\hat{N}>t) \leq 2 \mathbb{P}(\hat{L}>t), \quad \mathbb{P}(\hat{L}>3 t) \leq \mathbb{P}(\hat{N}>t)+4 \mathbb{P}^{2}(\hat{L}>t) .
$$

Suppose that $L<\infty$ a.s. We then have $\hat{L}<\infty$ a.s. and so by (3.1) we get $\hat{N}<\infty$ a.s. Hence, by the Borel-Cantelli lemma, there exists $r \geq 0$ such that $\hat{\Gamma}(r)<\infty$ and since $\lambda \Gamma(r+a) \leq \hat{\Gamma}(r)$, we have $N<\infty$ a.s. which proves the first implication in (a).

Suppose that $\mathbb{E} L<\infty$. Then we have $L<\infty$ a.s. and $\mathbb{E} \hat{L}<\infty$ and so by (3.1), we have $\mathbb{E} \hat{N}<\infty$. Hence, by (b) there exists $r>0$ such that $\int_{r}^{\infty} \hat{\Gamma}(t) d t<\infty$ and since

$$
\lambda \mathbb{P}(N>t+a) \leq \lambda \Gamma(t+a) \leq \hat{\Gamma}(t),
$$

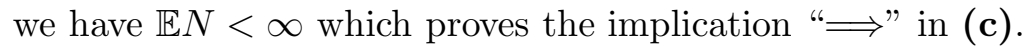

Suppose that $L<\infty$ a.s. and $\mathbb{E} N<\infty$. Then we have $\mathbb{E} \hat{N}<\infty$ and $\hat{L}<\infty$ a.s. So by (3.1) and a standard argument (see [6]) we have $\mathbb{E} \hat{L}<\infty$. By Lemma 3.1 (i), we have $\mathbb{E}\left\|T_{n}-\mathbb{E} T_{n}\right\| \leq$ $\mathbb{E}\left\|\hat{T}_{n}\right\|, n \geq 1$ and so we have

$$
\sum_{n=1}^{\infty} a_{n} \mathbb{E}\left\|T_{n}-\mathbb{E} T_{n}\right\| \leq \sum_{n=1}^{\infty} a_{n} \mathbb{E}\left\|\hat{T}_{n}\right\|=\mathbb{E} \hat{L}<\infty
$$

In particular, we have

$$
\sum_{n=1}^{\infty} a_{n}\left\|T_{n}-\mathbb{E} T_{n}\right\|<\infty \text { a.s. }
$$

and since $L=\sum_{n=1}^{\infty} a_{n}\left\|T_{n}\right\|<\infty$ a.s., we have $\sum_{n=1}^{\infty} a_{n}\left\|\mathbb{E} T_{n}\right\|<\infty$. Since $\mathbb{E}\left\|T_{n}\right\| \leq \mathbb{E}\left\|T_{n}-\mathbb{E} T_{n}\right\|+$ $\left\|\mathbb{E} T_{n}\right\|, n \geq 1$, we have $\mathbb{E} L=\sum_{n=1}^{\infty} a_{n} \mathbb{E}\left\|T_{n}\right\|<\infty$ which proves the implication "£" in (c).

Suppose that $\mathbb{E} L<\infty$. Then we have $\mathbb{E}\left\|V_{n}\right\|<\infty$ and by Lemma 3.1 (i), we have $\mathbb{E}\left\|T_{n}-\mathbb{E} T_{n}\right\| \leq$ $\mathbb{E}\left\|T_{i}-\mathbb{E} T_{i}\right\| \leq 2 \mathbb{E}\left\|T_{i}\right\|$ for all $1 \leq n \leq i$. Hence, we have

$$
b_{n} \mathbb{E}\left\|T_{n}-\mathbb{E} T_{n}\right\|=\sum_{i=n}^{\infty} a_{i} \mathbb{E}\left\|T_{n}-\mathbb{E} T_{n}\right\| \leq 2 \sum_{i=n}^{\infty} a_{i} \mathbb{E}\left\|T_{i}\right\|
$$

and since $\sum_{i=1}^{\infty} a_{i} \mathbb{E}\left\|T_{i}\right\|=\mathbb{E} L<\infty$, we see that $b_{n} \mathbb{E}\left\|T_{n}-\mathbb{E} T_{n}\right\| \rightarrow 0$ as $n \rightarrow \infty$.

Since $\sum_{n=1}^{\infty} b_{n}=\sum_{n=1}^{\infty} n a_{n}$, we see that (e) holds. 


\section{Proofs of Theorems 2.1, 2.2, and 2.4}

Let $\left\{X_{n} ; n \geq 1\right\}$ be a sequence of independent copies of a $\mathbf{B}$-valued random variable $X$. We consider a special case of Theorem 3.1 which will be used in the proofs of Theorems 2.1-2.4 and Corollary 5.1. Set $V_{n}=X_{n}, T_{n}=S_{n}, n \geq 1$. For given $0<p<2$, write

$$
G(t)=\mathbb{P}(\|X\|>t) \text { for } t \geq 0 \text { and } a_{n}=n^{-1 / p}-(n+1)^{-1 / p}, n \geq 1 .
$$

Then the hypotheses of Theorem 3.1 hold and by the mean value theorem we have

$$
\left(p^{-1} 2^{-1-1 / p}\right) n^{-1-1 / p}=p^{-1}(2 n)^{-1-1 / p} \leq p^{-1}(n+1)^{-1-1 / p} \leq a_{n} \leq\left(p^{-1}\right) n^{-1-1 / p}, \quad n \geq 1 .
$$

Let us define

$$
\Lambda_{p}(x)=\sum_{n=0}^{\infty}(n+1)^{-1 / p} x^{n}, \quad 0 \leq x \leq 1 .
$$

By partial summation, we have

$$
(1-x) \Lambda_{p}(x)=\sum_{n=1}^{\infty}\left(1-x^{n}\right) a_{n}
$$

and since

$$
\mathbb{P}\left(\max _{1 \leq i \leq n}\left\|X_{i}\right\|>t\right)=1-(1-G(t))^{n}
$$

we have

$$
\begin{aligned}
\sum_{n=1}^{\infty} a_{n} \mathbb{E}\left(\max _{1 \leq i \leq n}\left\|X_{i}\right\|\right) & =\sum_{n=1}^{\infty} a_{n} \int_{0}^{\infty}\left(1-(1-G(t))^{n}\right) d t \\
& =\int_{0}^{\infty}\left(\sum_{n=1}^{\infty} a_{n}\left(1-(1-G(t))^{n}\right)\right) d t \\
& =\int_{0}^{\infty} G(t) \Lambda_{p}(1-G(t)) d t .
\end{aligned}
$$

Adopting the notation of Theorem 3.1, we have

$$
b_{n}=n^{-1 / p}, n \geq 1 \text { and } \Gamma(t)=\sum_{n=1}^{\infty} \mathbb{P}\left(\|X\|>t n^{1 / p}\right) .
$$

Let $r>0$ be given. Since, for every real-valued random variable $U, \mathbb{E}(U-r)^{+}=\int_{r}^{\infty} \mathbb{P}(U>t) d t$, we have

$$
\begin{aligned}
\int_{r}^{\infty} \Gamma(t) d t & =\sum_{n=1}^{\infty} \int_{r}^{\infty} \mathbb{P}\left(\|X\|>t n^{1 / p}\right) d t \\
& =\sum_{n=1}^{\infty} \mathbb{E}\left(\frac{\|X\|}{n^{1 / p}}-r\right)^{+} \\
& =r \mathbb{E}\left(\sum_{n=1}^{\infty}\left(\frac{\|X\|}{r n^{1 / p}}-1\right)^{+}\right) \\
& =r \mathbb{E} \phi_{p}\left(\frac{1}{r}\|X\|\right)
\end{aligned}
$$


where

$$
\phi_{p}(s)=\sum_{n=1}^{\infty}\left(s n^{-1 / p}-1\right)^{+}=\sum_{1 \leq n \leq s^{p}}\left(s n^{-1 / p}-1\right), \quad s \geq 0 .
$$

Clearly, $\phi_{p}(s)$ is nondecreasing such that $\phi_{p}(s)=0$ for all $0 \leq s \leq 1$ and $\phi_{p}(s)>0$ for all $s>1$. Let

$$
\psi_{p}(s)= \begin{cases}s^{p}, & \text { if } 1<p<2 \\ s \ln (1+s), & \text { if } p=1 \\ s, & \text { if } 0<p<1\end{cases}
$$

It is easy to see that

$$
\lim _{s \rightarrow \infty} \frac{\phi_{p}(s)}{\psi_{p}(s)}= \begin{cases}1 /(p-1), & \text { if } 1<p<2 \\ 1, & \text { if } p=1 \\ \sum_{n=1}^{\infty} n^{-1 / p}, & \text { if } 0<p<1 .\end{cases}
$$

Thus, there exist positive constants $0<c_{p}<C_{p}$ satisfying

$$
c_{p} \psi_{p}(s) \leq \phi_{p}(s) \leq C_{p} \psi_{p}(s) \text { for all } s \geq 2 \text {. }
$$

By Lemma 3.1 (ii), we have for $n \geq 1$,

$$
\begin{aligned}
\mathbb{E}\left(\max _{1 \leq i \leq n}\left\|X_{i}\right\|\right) & \leq\|\mathbb{E} X\|+\mathbb{E}\left(\max _{1 \leq i \leq n}\left\|X_{i}-\mathbb{E} X\right\|\right) \\
& \leq\|\mathbb{E} X\|+4 \mathbb{E}\left\|S_{n}-\mathbb{E} S_{n}\right\| \\
& \leq\|\mathbb{E} X\|+8 \mathbb{E}\left\|S_{n}\right\| .
\end{aligned}
$$

Hence, by (4.2), (4.3), and Theorems 3.1 and 1.1, we obtain the following conclusions:

$$
\sum_{n=1}^{\infty} a_{n}\left\|S_{n}\right\|<\infty \text { a.s. } \Longrightarrow \sup _{n \geq 1} n^{-1 / p}\left\|X_{n}\right\|<\infty \text { a.s. } \Longleftrightarrow \mathbb{E}\|X\|^{p}<\infty .
$$

(C2) $\mathbb{E}\left(\sup _{n \geq 1} n^{-1 / p}\left\|X_{n}\right\|\right)<\infty \Longleftrightarrow \mathbb{E} \psi_{p}(\|X\|)<\infty \Longrightarrow \mathbb{E}\|X\|<\infty$.

(C3) $\sum_{n=1}^{\infty} a_{n} \mathbb{E}\left\|S_{n}\right\|<\infty \Longleftrightarrow \sum_{n=1}^{\infty} a_{n}\left\|S_{n}\right\|<\infty$ a.s. and $\mathbb{E} \psi_{p}(\|X\|)<\infty$.

(C4) $1 \leq p<2$ and $\sum_{n=1}^{\infty} a_{n}\left\|S_{n}\right\|<\infty$ a.s. $\Longrightarrow \mathbb{E}\|X\|<\infty$ and $\mathbb{E} X=0$.

(C5) If $\sum_{n=1}^{\infty} a_{n} \mathbb{E}\left\|S_{n}\right\|<\infty$, then $\int_{0}^{\infty} G(t) \Lambda_{p}(1-G(t)) d t<\infty$ and $\frac{S_{n}}{n^{1 / p}} \rightarrow 0$ a.s. and in $L^{1}(\mathbb{P})$.

Proof of Theorem 2.1 By (4.1), (C1), and (C3), we see that (2.1) and (2.2) are equivalent. For the given $1<p<2$, by (2.5) in the proof of Lemma 2 in Hechner and Heinkel [4], there exists a constant $0<A_{p}<1$ such that

$$
A_{p} x^{1 / p-1} \leq \Lambda_{p}(1-x) \leq x^{1 / p-1} \text { for all } 0<x \leq 1 .
$$

Hence, we see that the last part of Theorem 2.1 follows from (C1), (C4), and (C5). 
Proof of Theorem 2.2 The theorem is an immediate consequence of (C3) and (C4).

Proof of Theorem 2.4 First, by (4.1) and (C1), (2.17) follows from (2.16).

Conversely, suppose that (2.17) holds. By (4.1) and Theorem 3.1, we have

$$
\left(p^{-1} 2^{-1-1 / p}\right) \sum_{n=1}^{\infty} \frac{1}{n}\left(\frac{\left\|S_{n}\right\|}{n^{1 / p}}\right) \leq \sum_{n=1}^{\infty} a_{n}\left\|S_{n}\right\| \leq \sum_{n=1}^{\infty} n^{-1 / p}\left\|X_{n}\right\| .
$$

Since $0<p<1$, by $(2.17)$ we have

$$
\sum_{n=1}^{\infty} n^{-1 / p}\left\|X_{n}\right\|<\infty \text { a.s. }
$$

(see Theorem 5.1.3 in Chow and Teicher [2, p. 115]) and (2.16) follows.

Furthermore, for $0<p<1$, the equivalence of $(2.18)$ and $(2.19)$ is trivial since

$$
\mathbb{E}\|X\| \leq \sum_{n=1}^{\infty} \frac{1}{n}\left(\frac{\mathbb{E}\left\|S_{n}\right\|}{n^{1 / p}}\right) \leq\left(\sum_{n=1}^{\infty} \frac{1}{n^{1 / p}}\right) \mathbb{E}\|X\|<\infty .
$$

The proof of Theorem 2.4 is complete.

\section{Proofs of Theorem 2.3 and Corollaries 2.2 and 2.3}

Let $\left\{X_{n} ; n \geq 1\right\}$ be a sequence of independent copies of the $\mathbf{B}$-valued random variable $X$. Write

$$
X_{n}^{(1)}=X_{n} I\left\{\left\|X_{n}\right\| \leq n\right\} \text { and } X_{n}^{(2)}=X_{n} I\left\{\left\|X_{n}\right\|>n\right\}, \quad n \geq 1
$$

and

$$
S_{n}^{(1)}=\sum_{k=1}^{n} X_{k}^{(1)} \text { and } S_{n}^{(2)}=\sum_{k=1}^{n} X_{k}^{(2)}, \quad n \geq 1
$$

For the proof of Theorem 2.3, we need the following four preliminary lemmas.

Lemma 5.1. Let $X$ be a $\mathbf{B}$-valued random variable with $\mathbb{E}\|X\|<\infty$. Then

$$
\sum_{n=1}^{\infty} \frac{1}{n^{2}} \sum_{k=1}^{n} \mathbb{E}\|X\| I\{k<\|X\| \leq n\}<\infty
$$

and

$$
\sum_{n=1}^{\infty} \frac{u_{n}}{n^{2}}<\infty
$$


Proof Since $\mathbb{E}\|X\|<\infty$, we have that

$$
\begin{aligned}
\sum_{n=1}^{\infty} \frac{1}{n^{2}} \sum_{k=1}^{n} \mathbb{E}\|X\| I\{k<\|X\| \leq n\} & =\sum_{k=1}^{\infty} \sum_{n=k}^{\infty} \frac{1}{n^{2}} \mathbb{E}\|X\| I\{k<\|X\| \leq n\} \\
& \leq \sum_{k=1}^{\infty} \sum_{n=k}^{\infty} \frac{1}{n^{2}} \sum_{m=k}^{n} m \mathbb{P}(m-1<\|X\| \leq m) \\
& =\sum_{k=1}^{\infty} \sum_{m=k}^{\infty} m\left(\sum_{n=m}^{\infty} \frac{1}{n^{2}}\right) \mathbb{P}(m-1<\|X\| \leq m) \\
& \leq \sum_{k=1}^{\infty} \sum_{m=k}^{\infty} m \times \frac{2}{m} \mathbb{P}(m-1<\|X\| \leq m) \\
& =2 \sum_{m=1}^{\infty} \sum_{k=1}^{m} \mathbb{P}(m-1<\|X\| \leq m) \\
& =2 \sum_{m=1}^{\infty} m \mathbb{P}(m-1<\|X\| \leq m) \\
& <\infty
\end{aligned}
$$

proving (5.1).

We now show that (5.2) follows from $\mathbb{E}\|X\|<\infty$. Since $\left\{u_{n} ; n \geq 1\right\}$ is an increasing sequence with

$$
\sup _{n \geq 1} u_{n}=\sup \{x: \mathbb{P}(\mid X \| \leq x)<1\}
$$

we obtain that

$$
\begin{aligned}
\sum_{n=1}^{\infty} \frac{u_{n+1}-u_{n}}{n} & \leq \sum_{n=1}^{\infty} 2 \int_{u_{n}}^{u_{n+1}} \mathbb{P}(\|X\|>x) d x \\
& =2 \int_{u_{1}}^{\infty} \mathbb{P}(\|X\|>x) d x \\
& \leq 2 \mathbb{E}\|X\|<\infty .
\end{aligned}
$$

Note that for each integer $m \geq 2$, partial summation yields

$$
\begin{aligned}
\sum_{n=1}^{m} \frac{u_{n+1}-u_{n}}{n} & =\left(\frac{u_{m+1}}{m}-u_{1}\right)+\sum_{n=2}^{m}\left(\frac{1}{n-1}-\frac{1}{n}\right) u_{n} \\
& \geq-2 u_{1}+\sum_{n=1}^{m} \frac{u_{n}}{n^{2}} .
\end{aligned}
$$

We thus see that

$$
\sum_{n=1}^{m} \frac{u_{n}}{n^{2}} \leq 2 u_{1}+\sum_{n=1}^{\infty} \frac{u_{n+1}-u_{n}}{n}<\infty
$$


proving (5.2).

Lemma 5.2. Let $\left\{X_{n} ; n \geq 1\right\}$ be be a sequence of independent copies of $\mathbf{B}$-valued random variable $X$ with $\mathbb{E}\|X\|<\infty$. Write

$$
U_{n}=\sum_{k=1}^{n} X_{k} I\left\{\left\|X_{k}\right\| \leq n\right\}, n \geq 1 .
$$

Then

$$
\begin{aligned}
& \text { (i) } \sum_{n=1}^{\infty} \frac{1}{n}\left(\frac{\left\|\mathbb{E} S_{n}^{(1)}\right\|}{n}\right)<\infty \text { if and only if }(2.12) \text { holds, } \\
& \text { (ii) } \sum_{n=1}^{\infty} \frac{1}{n}\left(\frac{\mathbb{E}\left\|S_{n}^{(1)}-\mathbb{E} S_{n}^{(1)}\right\|}{n}\right)<\infty \text { if and only if } \sum_{n=1}^{\infty} \frac{1}{n}\left(\frac{\mathbb{E}\left\|U_{n}-\mathbb{E} U_{n}\right\|}{n}\right)<\infty .
\end{aligned}
$$

Proof Note that by (5.1) of Lemma 5.1,

$$
\begin{aligned}
& \sum_{n=1}^{\infty} \frac{1}{n}\left\|\frac{\mathbb{E} S_{n}^{(1)}}{n}-\mathbb{E} X I\{\|X\| \leq n\}\right\| \\
& =\sum_{n=1}^{\infty} \frac{1}{n}\left\|\frac{\sum_{k=1}^{n}(\mathbb{E} X I\{\|X\| \leq k\}-\mathbb{E} X I\{\|X\| \leq n\})}{n}\right\| \\
& \leq \sum_{n=1}^{\infty} \frac{1}{n^{2}} \sum_{k=1}^{n} \mathbb{E}\|X\| I\{k<\|X\| \leq n\} \\
& <\infty
\end{aligned}
$$

Thus part (i) follows.

Similarly, by (5.1) of Lemma 5.1,

$$
\begin{aligned}
& \sum_{n=1}^{\infty} \frac{1}{n}\left(\frac{\mathbb{E}\left\|\left(S_{n}^{(1)}-\mathbb{E} S_{n}^{(1)}\right)-\left(U_{n}-\mathbb{E} U_{n}\right)\right\|}{n}\right) \\
& \leq 2 \sum_{n=1}^{\infty} \frac{1}{n^{2}} \sum_{k=1}^{n} \mathbb{E}\|X\| I\{k<\|X\| \leq n\} \\
& <\infty
\end{aligned}
$$

and part (ii) follows.

The proof of the next lemma is similar to that of Lemma 4 of Hechner and Heinkel [4] and contains a nice application of Lemma 1 of Hechner and Heinkel [4].

Lemma 5.3. Let $\mathbf{B}$ be a Banach space of stable type 1 . Let $\left\{X_{n} ; n \geq 1\right\}$ be a sequence of independent copies of a $\mathbf{B}$-valued random variable $X$ with $\mathbb{E}\|X\|<\infty$. Write

$$
U_{n}^{(1)}=\sum_{k=1}^{n} X_{k} I\left\{\left\|X_{k}\right\| \leq u_{n}\right\}, n \geq 1 .
$$


Then

$$
\sum_{n=1}^{\infty} \frac{1}{n}\left(\frac{\mathbb{E}\left\|U_{n}^{(1)}-\mathbb{E} U_{n}^{(1)}\right\|}{n}\right)<\infty .
$$

Proof Since B is of stable type 1, the Maurey-Pisier [12] theorem asserts that it is also of stable type $q$ for some $1<q<2$. Applying Lemma 1 of Hechner and Heinkel [4], there exists a universal constant $0<c(q)<\infty$ such that

$$
\begin{aligned}
\mathbb{E}\left\|U_{n}^{(1)}-\mathbb{E} U_{n}^{(1)}\right\| & \leq c(q)\left(\sup _{t>0} t^{q} \sum_{k=1}^{n} \mathbb{P}\left(\left\|X_{k}\right\| I\left\{\left\|X_{k}\right\| \leq u_{n}\right\}>t\right)\right)^{1 / q} \\
& \leq c(q)\left(n \sup _{0 \leq t \leq u_{n}} t^{q} \mathbb{P}(\|X\|>t)\right)^{1 / q}, \quad n \geq 1 .
\end{aligned}
$$

It is easy to see that for all $x>0$,

$$
\begin{aligned}
\left(\int_{0}^{x} \mathbb{P}^{1 / q}(\|X\|>t) d t\right)^{q} & \geq\left(\int_{0}^{x} \mathbb{P}^{1 / q}(\|X\|>x) d t\right)^{q} \\
& =x^{q} \mathbb{P}(\|X\|>x) .
\end{aligned}
$$

We thus have that

$$
\begin{aligned}
\mathbb{E}\left\|U_{n}^{(1)}-\mathbb{E} U_{n}^{(1)}\right\| & \leq c(q)\left(n \sup _{0 \leq t \leq u_{n}} t^{q} \mathbb{P}(\|X\|>t)\right)^{1 / q} \\
& \leq c(q) n^{1 / q} \int_{0}^{u_{n}} \mathbb{P}^{1 / q}(\|X\|>t) d t, \quad n \geq 1 .
\end{aligned}
$$

Let $u_{0}=0$ and note that $\mathbb{P}(\|X\|>t) \geq 1 / k$ for $t \in\left[u_{k-1}, u_{k}\right), k \geq 1$. It follows that

$$
\begin{aligned}
\sum_{n=1}^{\infty} \frac{1}{n}\left(\frac{\mathbb{E}\left\|U_{n}^{(1)}-\mathbb{E} U_{n}^{(1)}\right\|}{n}\right) & \leq c(q) \sum_{n=1}^{\infty} \frac{1}{n^{2-1 / q}} \int_{0}^{u_{n}} \mathbb{P}^{1 / q}(\|X\|>t) d t \\
& =c(q) \sum_{n=1}^{\infty} \frac{1}{n^{2-1 / q}} \sum_{k=1}^{n} \int_{u_{k-1}}^{u_{k}} \mathbb{P}^{1 / q}(\|X\|>t) d t \\
& =c(q) \sum_{k=1}^{\infty}\left(\sum_{n=k}^{\infty} \frac{1}{n^{2-1 / q}}\right) \int_{u_{k-1}}^{u_{k}} \mathbb{P}^{1 / q}(\|X\|>t) d t \\
& \leq C \sum_{k=1}^{\infty} \frac{1}{k^{1-1 / q}} \int_{u_{k-1}}^{u_{k}} \mathbb{P}^{1 / q}(\|X\|>t) d t \\
& \leq C \int_{0}^{\infty} \mathbb{P}(\|X\|>t) d t \\
& =C \mathbb{E}\|X\|<\infty
\end{aligned}
$$

proving (5.3) and completing the proof of Lemma 5.3. 
Lemma 5.4. Let $Y_{1}, Y_{2}, \ldots, Y_{n}$ be i.i.d. nonnegative real-valued random variables such that

$$
\mathbb{P}\left(Y_{1}>0\right) \leq \frac{1}{n}
$$

Then

$$
\mathbb{E}\left(\max _{1 \leq k \leq n} Y_{k}\right) \geq \frac{n}{2} \mathbb{E} Y_{1} .
$$

Proof Since $Y_{1}, Y_{2}, \ldots, Y_{n}$ are i.i.d. nonnegative real-valued random variables, we have

$$
\mathbb{P}\left(\max _{1 \leq k \leq n} Y_{k}>t\right)=1-\left(1-\mathbb{P}\left(Y_{1}>t\right)\right)^{n} \geq 1-e^{-n \mathbb{P}\left(Y_{1}>t\right)} \text { for all } t \geq 0
$$

Since $\left(1-e^{-x}\right) / x$ is decreasing, we have $1-e^{-x} \geq \beta x$ for all $0 \leq x \leq 1$ where $\beta=1-e^{-1} \geq 1 / 2$. Hence, it follows from (5.4) that

$$
\mathbb{P}\left(\max _{1 \leq k \leq n} Y_{k}>t\right) \geq \frac{n}{2} \mathbb{P}\left(Y_{1}>t\right) \quad \text { for all } t>0,
$$

which ensures (5.5).

Proof of Theorem 2.3 (Sufficiency) Since

$$
\left\|S_{n}\right\| \leq\left\|\mathbb{E} S_{n}^{(1)}\right\|+\left\|S_{n}^{(1)}-\mathbb{E} S_{n}^{(1)}\right\|+\left\|S_{n}^{(2)}\right\|, n \geq 1,
$$

(2.10) will follow if we can show that (2.11), (2.12), and (2.13) imply

$$
\begin{gathered}
\sum_{n=1}^{\infty} \frac{1}{n}\left(\frac{\left\|\mathbb{E} S_{n}^{(1)}\right\|}{n}\right)<\infty, \\
\sum_{n=1}^{\infty} \frac{1}{n}\left(\frac{\mathbb{E}\left\|S_{n}^{(1)}-\mathbb{E} S_{n}^{(1)}\right\|}{n}\right)<\infty,
\end{gathered}
$$

and

$$
\sum_{n=1}^{\infty} \frac{1}{n}\left(\frac{\left\|S_{n}^{(2)}\right\|}{n}\right)<\infty \text { a.s. }
$$

By Lemma 5.2 (i), (5.6) follows from (2.11) and (2.12). Since

$$
\sum_{n=1}^{\infty} \mathbb{P}\left(X_{n}^{(2)} \neq 0\right)=\sum_{n=1}^{\infty} \mathbb{P}(\|X\|>n) \leq \mathbb{E}\|X\|<\infty
$$

by the Borel-Cantelli lemma we have that

$$
\mathbb{P}\left(X_{n}^{(2)} \neq 0 \text { i.o. }(n)\right)=0
$$

which ensures that

$$
\left\|S_{n}^{(2)}\right\|=O(1) \quad \text { a.s. as } n \rightarrow \infty \text {. }
$$

Thus (5.8) holds. 
We now show that (2.11) and (2.13) imply (5.7). Since $\mathbb{E}\|X\|<\infty$, by Lemma 5.2 (ii), (5.7) is equivalent to

$$
\sum_{n=1}^{\infty} \frac{1}{n}\left(\frac{\mathbb{E}\left\|U_{n}-\mathbb{E} U_{n}\right\|}{n}\right)<\infty
$$

Now (2.9) holds recalling the implication $((2.8) \Rightarrow(2.9))$. Hence we can assume, without loss of generality, that $u_{n}<n$ for all $n \geq 1$. Write

$$
U_{n}^{(2)}=\sum_{k=1}^{n} X_{k} I\left\{u_{n}<\left\|X_{k}\right\| \leq n\right\}, n \geq 1 .
$$

Clearly, (5.9) will follow provided we can show

$$
\sum_{n=1}^{\infty} \frac{1}{n}\left(\frac{\mathbb{E}\left\|U_{n}^{(1)}-\mathbb{E} U_{n}^{(1)}\right\|}{n}\right)<\infty
$$

and

$$
\sum_{n=1}^{\infty} \frac{1}{n}\left(\frac{\mathbb{E}\left\|U_{n}^{(2)}-\mathbb{E} U_{n}^{(2)}\right\|}{n}\right)<\infty
$$

Since $\mathbb{E}\|X\|<\infty$ and $\mathbf{B}$ is of stable type 1 , by Lemma $5.3,(5.10)$ holds. Note that, for all $n \geq 1$,

$$
\begin{aligned}
\mathbb{E}\left\|U_{n}^{(2)}-\mathbb{E} U_{n}^{(2)}\right\| & \leq \sum_{k=1}^{n} \mathbb{E}\left\|X_{k} I\left\{u_{n}<\left\|X_{k}\right\| \leq n\right\}-\mathbb{E} X_{k} I\left\{u_{n}<\left\|X_{k}\right\| \leq n\right\}\right\| \\
& \leq 2 n \mathbb{E}\|X\| I\left\{u_{n}<\|X\| \leq n\right\} \\
& =2 n \int_{u_{n}}^{n} t d \mathbb{P}(\|X\| \leq t) \\
& \leq 2 n u_{n} \mathbb{P}\left(\|X\|>u_{n}\right)+2 n \int_{u_{n}}^{n} \mathbb{P}(\|X\|>t) d t \\
& \leq 2 u_{n}+2 n \int_{u_{n}}^{n} \mathbb{P}(\|X\|>t) d t
\end{aligned}
$$

Now (5.2) holds by Lemma 5.1. Thus (5.11) follows from (5.2) and (2.13). The proof of the sufficiency half of Theorem 2.3 is complete.

Proof of Theorem 2.3 (Necessity) First, by (4.1) with $p=1,(2.10)$ is equivalent to

$$
\sum_{n=1}^{\infty} a_{n}\left\|S_{n}\right\|<\infty \text { a.s. }
$$

Thus, by (C4) with $p=1$, (2.11) follows. In particular, we have $\mathbb{E}\|X\|<\infty$. Again by (4.1) with $p=1$ and arguing as in the proof of (5.8) in the sufficiency half, we have

$$
\sum_{n=1}^{\infty} a_{n}\left\|S_{n}^{(2)}\right\|<\infty \text { a.s. }
$$


Note that

$$
\left\|S_{n}^{(1)}\right\| \leq\left\|S_{n}\right\|+\left\|S_{n}^{(2)}\right\|, \quad n \geq 1 .
$$

It thus follows from (2.10) that

$$
\sum_{n=1}^{\infty} a_{n}\left\|S_{n}^{(1)}\right\|<\infty \text { a.s. }
$$

Since, with $p=1$,

$$
N=\sup _{n \geq 1} b_{n}\left\|X_{n}^{(1)}\right\|=\sup _{n \geq 1} n^{-1}\left\|X_{n}^{(1)}\right\| \leq 1,
$$

it follows from (5.12) and Theorem 3.1 (c) that

$$
\sum_{n=1}^{\infty} a_{n} \mathbb{E}\left\|S_{n}^{(1)}\right\|<\infty
$$

which by (4.1) ensures that

$$
\sum_{n=1}^{\infty} \frac{1}{n}\left(\frac{\mathbb{E}\left\|S_{n}^{(1)}\right\|}{n}\right)<\infty
$$

Note that (5.13) implies

$$
\sum_{n=1}^{\infty} \frac{1}{n}\left(\frac{\left\|\mathbb{E} S_{n}^{(1)}\right\|}{n}\right)<\infty
$$

which by $\mathbb{E}\|X\|<\infty$ and Lemma 5.2 (i) yields (2.12). Moreover, by Lemma 5.2 (ii), (5.13) then ensures that

$$
\sum_{n=1}^{\infty} \frac{1}{n}\left(\frac{\mathbb{E}\left\|U_{n}-\mathbb{E} U_{n}\right\|}{n}\right)<\infty .
$$

Since $\left\|U_{n}^{(2)}-\mathbb{E} U_{n}^{(2)}\right\| \leq\left\|U_{n}-\mathbb{E} U_{n}\right\|+\left\|U_{n}^{(1)}-\mathbb{E} U_{n}^{(1)}\right\|, n \geq 1$ and $\mathbf{B}$ is of stable type 1 , it follows from Lemma 5.3 and (5.14) that

$$
\sum_{n=1}^{\infty} \frac{1}{n}\left(\frac{\mathbb{E}\left\|U_{n}^{(2)}-\mathbb{E} U_{n}^{(2)}\right\|}{n}\right)<\infty
$$

By Lemma 3.1 (ii),

$$
\mathbb{E} \max _{1 \leq k \leq n}\left\|X_{k} I\left\{u_{n}<\left\|X_{k}\right\| \leq n\right\}-\mathbb{E} X I\left\{u_{n}<\|X\| \leq n\right\}\right\| \leq 4 \mathbb{E}\left\|U_{n}^{(2)}-\mathbb{E} U_{n}^{(2)}\right\|, n \geq 1 .
$$

It thus follows from (5.15) and $\mathbb{E}\|X\|<\infty$ that

$$
\begin{aligned}
& \sum_{n=1}^{\infty} \frac{1}{n}\left(\frac{\mathbb{E} \max _{1 \leq k \leq n}\left\|X_{k} I\left\{u_{n}<\left\|X_{k}\right\| \leq n\right\}\right\|}{n}\right) \\
& \leq \sum_{n=1}^{\infty} \frac{1}{n}\left(\frac{4 \mathbb{E}\left\|U_{n}^{(2)}-\mathbb{E} U_{n}^{(2)}\right\|}{n}\right)+\sum_{n=1}^{\infty} \frac{\mathbb{E}\|X\|}{n^{2}} \\
& <\infty,
\end{aligned}
$$


and hence, by Lemma 5.4, noting that $\mathbb{P}\left(\|X\|>u_{n}\right) \leq n^{-1}, n \geq 1$, we get that

$$
\sum_{n=1}^{\infty} \frac{\mathbb{E}\|X\| I\left\{u_{n}<\|X\| \leq n\right\}}{n}<\infty .
$$

Using partial integration, one can easily see that

$$
\begin{aligned}
& \left|\mathbb{E}\|X\| I\left\{u_{n}<\|X\| \leq n\right\}-\int_{u_{n}}^{n} \mathbb{P}(\|X\|>t) d t\right| \\
& \leq \frac{u_{n}}{n}+n \mathbb{P}(\|X\|>n), \quad n \geq 1 .
\end{aligned}
$$

Since $\mathbb{E}\|X\|<\infty$, we have

$$
\sum_{n=1}^{\infty} \frac{n \mathbb{P}(\|X\|>n)}{n}=\sum_{n=1}^{n} \mathbb{P}(\|X\|>n)<\infty,
$$

and, by Lemma 5.1, (5.2) holds. We thus see that (2.13) follows from (5.16), (5.17), (5.18), and (5.2) thereby completing the proof of the necessity half of Theorem 2.3.

Lemma 5.5. Let $X$ be a $\mathbf{B}$-valued random variable such that

$$
\mathbb{E} X=0 \text { and } \mathbb{E}\|X\| \ln (1+\|X\|)<\infty .
$$

Then (2.12) holds.

Proof Argue as in Exercise 5.1.6 (ii) of Chow and Teicher [2, p. 123]. The details are left to the reader.

Lemma 5.6. Let $X$ be a $\mathbf{B}$-valued random variable such that

$$
\mathbb{E}\|X\| \ln ^{\delta}(1+\|X\|)<\infty \text { for some } \delta>0 .
$$

Then (2.13) holds.

Proof It follows from (5.17) that, under the condition $\mathbb{E}\|X\|<\infty,(2.13)$ and (5.16) are equivalent. We thus only need to show that (5.19) implies (5.16). To this end, let $q=(2+\delta) / 2$. Applying Hölder's inequality, we have that

$$
\begin{aligned}
& \mathbb{E}\|X\| I\left\{u_{n}<\|X\| \leq n\right\} \\
& =\mathbb{E}\left(\|X\| I\left\{u_{n}<\|X\| \leq n\right\}\right)\left(I\left\{u_{n}<\|X\| \leq n\right\}\right) \\
& \leq\left(\mathbb{E}\left(\|X\| I\left\{u_{n}<\|X\| \leq n\right\}\right)^{q}\right)^{1 / q}\left(\mathbb{E}\left(I\left\{u_{n}<\|X\| \leq n\right\}\right)^{q /(q-1)}\right)^{(q-1) / q} \\
& \leq\left(\mathbb{E}(\|X\| I\{\|X\| \leq n\})^{q}\right)^{1 / q}\left(\mathbb{E} I\left\{\|X\|>u_{n}\right\}\right)^{(q-1) / q} \\
& =\left(\mathbb{E}(\|X\| I\{\|X\| \leq n\})^{q}\right)^{1 / q} \mathbb{P}^{(q-1) / q}\left(\|X\|>u_{n}\right) \\
& \leq \frac{\left(\mathbb{E}(\|X\| I\{\|X\| \leq n\})^{q}\right)^{1 / q}}{n^{1-1 / q}}, \quad n \geq 1 .
\end{aligned}
$$


Thus, (5.16) follows if we can show that (5.19) implies

$$
\sum_{n=1}^{\infty} \frac{\left(\mathbb{E}(\|X\| I\{\|X\| \leq n\})^{q}\right)^{1 / q}}{n^{2-1 / q}}<\infty
$$

Write

$$
f_{n}=\frac{\left(\ln ^{\delta / q}(1+n)\right)\left(\mathbb{E}\|X\|^{q} I\{\|X\| \leq n\}\right)^{1 / q}}{n}, \quad g_{n}=\frac{1}{n^{1-1 / q} \ln ^{\delta / q}(1+n)}, n \geq 1 .
$$

Applying Hölder's inequality again, we have that

$$
\sum_{n=1}^{\infty} \frac{\left(\mathbb{E}\|X\|^{q} I\{\|X\| \leq n\}\right)^{1 / q}}{n^{2-1 / q}}=\sum_{n=1}^{\infty} f_{n} g_{n} \leq\left(\sum_{n=1}^{\infty} f_{n}^{q}\right)^{1 / q}\left(\sum_{n=1}^{\infty} g_{n}^{q /(q-1)}\right)^{(q-1) / q}
$$

It is easy to see that

$$
\sum_{n=1}^{\infty} g_{n}^{q /(q-1)}=\sum_{n=1}^{\infty} \frac{1}{n \ln ^{2}(1+n)}<\infty
$$

Since $\mathbb{E}\|X\| \ln ^{\delta}(1+\|X\|)<\infty$, we have that

$$
\begin{aligned}
\sum_{n=1}^{\infty} f_{n}^{q} & =\sum_{n=1}^{\infty} \frac{\left(\ln ^{\delta}(1+n)\right) \mathbb{E}\|X\|^{q} I\{\|X\| \leq n\}}{n^{q}} \\
& \leq \sum_{n=1}^{\infty} \frac{\ln ^{\delta}(1+n)}{n^{q}} \sum_{k=1}^{n} k^{q} \mathbb{P}(k-1<\|X\| \leq k) \\
& =\sum_{k=1}^{\infty}\left(\sum_{n=k}^{\infty} \frac{\ln ^{\delta}(1+n)}{n^{q}}\right) k^{q} \mathbb{P}(k-1<\|X\| \leq k) \\
& \leq C \sum_{k=1}^{\infty} \frac{\ln ^{\delta}(1+k)}{k^{q-1}} \times k^{q} \mathbb{P}(k-1<\|X\| \leq k) \\
& =C \sum_{k=1}^{\infty}\left(k \ln ^{\delta}(1+k)\right) \mathbb{P}(k-1<\|X\| \leq k) \\
& <\infty
\end{aligned}
$$

Thus (5.20) holds. This completes the proof of Lemma 5.6.

Proof of Corollary 2.2 By Theorem 2.2, we only need to show that, under the assumption that B is of stable type 1, (2.6) follows from (2.7). Clearly, (2.7) implies (2.11). Applying Lemmas 5.5 and 5.6, (2.12) and (2.13) follow from the second half of (2.7). Thus, by Theorem 2.3, (2.6) holds.

Proof of Corollary 2.3 The conclusion of Corollary 2.3 follows immediately from Lemma 5.6 and Theorem 2.3 . 
From the definition of $\Lambda_{1}(x)$ given in Section 4, we see that

$$
\Lambda_{1}(0)=1 \text { and } \Lambda_{1}(x)=-\frac{\ln (1-x)}{x} \text { for all } 0<x \leq 1 .
$$

Hence, by (C5) we see that (2.5) implies

$$
\int_{0}^{\infty} G(t) \ln \frac{1}{G(t)} d t<\infty
$$

Conversely, if (5.21) holds, then (2.14) holds and an easy computation shows that $\mathbb{E}\|X\| \ln (1+$ $\|X\|)<\infty$. So by Lemma 5.5, Lemma 5.6, Theorem 2.3, and Theorem 2.2 we obtain the following result.

Corollary 5.1. Let $\mathbf{B}$ be a Banach space of stable type 1 . Let $\left\{X_{n} ; n \geq 1\right\}$ be a sequence of independent copies of a $\mathbf{B}$-valued random variable $X$. Then we have

$$
\sum_{n=1}^{\infty} \frac{1}{n}\left(\frac{\mathbb{E}\left\|S_{n}\right\|}{n}\right)<\infty \text { if and only if } \mathbb{E} X=0 \text { and } \int_{0}^{\infty} G(t) \ln \frac{1}{G(t)} d t<\infty \text {. }
$$

For illustrating the conditions (2.11), (2.12), and (2.13) of Theorem 2.3, we now present the following three examples.

Example 5.1. Let $X$ be a real-valued random variable such that

$$
\mathbb{P}\left(X=-\frac{1}{1-a}\right)=1-a \text { and } \mathbb{P}(X>x)=\int_{x}^{\infty} \frac{1}{t^{2} \ln ^{2} t} d t, \quad x \geq e
$$

where $a=\int_{e}^{\infty} \frac{1}{t^{2} \ln ^{2} t} d t$. Then

$$
\mathbb{E} X=0, \quad \mathbb{E}|X| \ln ^{\delta}(1+|X|)<\infty \text { for all } 0<\delta<1,
$$

and, for all sufficiently large $n$,

$$
\mathbb{E} X I\{|X| \leq n\}=-\mathbb{E} X I\{|X|>n\}=-\int_{n}^{\infty} \frac{1}{t \ln ^{2} t} d t=-\frac{1}{\ln n}
$$

Note that

$$
\sum_{n=2}^{\infty} \frac{1}{n \ln n}=\infty
$$

Thus (2.12) fails but, by Lemma 5.6, (2.13) holds.

Example 5.2. Let $X$ be a real-valued symmetric random variable with density function

$$
f(x)=\frac{b}{x^{2}(\ln |x|)(\ln \ln |x|)^{2}} I\{|x|>3\},
$$

where $0<b<\infty$ is such that $\int_{-\infty}^{\infty} f(x) d x=1$. Clearly, (2.11) and (2.12) hold. Since

$$
\mathbb{P}(|X|>x) \sim \frac{2 b}{x(\ln x)(\ln \ln x)^{2}} \quad \text { as } x \rightarrow \infty,
$$


we see that

$$
u_{n} \sim \frac{2 b n}{(\ln n)(\ln \ln n)^{2}} \quad \text { as } n \rightarrow \infty
$$

and hence, for all sufficiently large $n$,

$$
\begin{aligned}
\int_{u_{n}}^{n} \mathbb{P}(|X|>t) d t & \geq \int_{\frac{b n}{(\ln n)(\ln \ln n)^{2}}}^{n} \frac{b}{t(\ln t)(\ln \ln t)^{2}} d t \\
& \geq \frac{b}{(\ln n)(\ln \ln n)^{2}} \int_{\frac{b n}{(\ln n)(\ln \ln n)^{2}}}^{n} \frac{1}{t} d t \\
& \sim \frac{b}{(\ln n)(\ln \ln n)} \text { as } n \rightarrow \infty
\end{aligned}
$$

Note that

$$
\sum_{n=3}^{\infty} \frac{b}{n(\ln n)(\ln \ln n)}=\infty
$$

and so (2.13) fails.

Example 5.3. Let $X$ be a real-valued symmetric random variable with density function

$$
f(x)=\frac{1}{2 x^{2}} I\{|x|>1\} .
$$

Clearly, (2.12) holds. Since

$$
\mathbb{P}(|X|>x)=\frac{1}{x}, \quad x>1
$$

we have that

$$
u_{n}=n, \quad n \geq 1 \text {. }
$$

Thus (2.13) also holds. However, (2.11) fails.

\section{Acknowledgments}

The authors are extremely grateful to the Referee for very carefully reading the manuscript and for offering numerous comments and suggestions which enabled us to substantially improve the paper. The Referee did not only provide comments to help us to improve the presentation but more significantly the Referee presented improved versions of some of the main results complete with their proofs. Specifically, the Referee significantly improved our original version of Theorem 3.1 and this improved version led to more elegant proofs of many other results in the paper as was pointed out to us by the Referee. The authors also thank Professor Andrzej Korzeniowski for his interest in our work and for pointing out to us the relevance of his paper [9] to ours. The research of Deli Li was partially supported by a grant from the Natural Sciences and Engineering Research Council of Canada and the research of Yongcheng Qi was partially supported by NSF Grant DMS-0604176. 


\section{References}

1. Azlarov, T. A., Volodin, N. A.: Laws of large numbers for identically distributed Banachspace valued random variables. Teor. Veroyatnost. i Primenen. 26, 584-590 (1981), in Russian. English translation in Theory Probab. Appl. 26, 573-580 (1981).

2. Chow, Y.S., Teicher, H.: Probability Theory: Independence, Interchangeability, Martingales, 3rd ed. Springer-Verlag, New York (1997).

3. de Acosta, A.: Inequalities for $B$-valued random vectors with applications to the law of large numbers. Ann. Probab. 9, 157-161 (1981).

4. Hechner, F., Heinkel, B.: The Marcinkiewicz-Zygmund LLN in Banach spaces: A generalized martingale approach. J. Theor. Probab. 23, 509-522 (2010).

5. Hoffmann-Jørgensen, J.: Sums of independent Banach space valued random variables. Studia Math. 52, 159-186 (1974).

6. Hoffmann-Jørgensen, J., Pisier, G.: The law of large numbers and the central limit theorem in Banach spaces. Ann. Probab. 4, 587-599 (1976).

7. Klass, M. J.: Properties of optimal extended-valued stopping rules for $S_{n} / n$. Ann. Probab. 1 719-757 (1973).

8. Kolmogoroff, A.: Sur la loi forte des grands nombres. C. R. Acad. Sci. Paris Sér. Math. 191, 910-912 (1930).

9. Korzeniowski, A.: On Marcinkiewicz SLLN in Banach spaces. Ann. Probab. 12, 279-280 (1984).

10. Ledoux, M., Talagrand, M.: Probability in Banach Spaces: Isoperimetry and Processes. Springer-Verlag, Berlin (1991).

11. Marcinkiewicz, J., Zygmund, A.: Sur les fonctions indépendantes. Fund. Math. 29, 60-90 (1937).

12. Maurey, B., Pisier, G.: Séries de variables aléatoires vectorielles indépendantes et propriétés géométriques des espaces de Banach. Studia Math. 58, 45-90 (1976).

13. Marcus, M. B., Woyczyński, W. A.: Stable measures and central limit theorems in spaces of stable type. Trans. Amer. Math. Soc. 251, 71-102 (1979).

14. Mourier, E.: Eléments aléatoires dans un espace de Banach. Ann. Inst. H. Poincaré 13, 161-244 (1953).

15. Pisier, G.: Probabilistic methods in the geometry of Banach spaces, in Probability and Analysis, Lectures given at the 1st 1985 Session of the Centro Internazionale Matematico Estivo (C.I.M.E.), Lecture Notes in Mathematics, Vol. 1206, 167-241, Springer-Verlag, Berlin (1986).

16. Rosiński, J.: Remarks on Banach spaces of stable type. Probab. Math. Statist. 1, 67-71 (1980). 
17. Taylor, R. L.: Stochastic Convergence of Weighted Sums of Random Elements in Linear Spaces, Lecture Notes in Mathematics, Vol. 672, Springer-Verlag, Berlin (1978).

18. Woyczyński, W. A.: Geometry and martingales in Banach spaces-Part II: Independent increments, in Probability on Banach Spaces (Edited by J. Kuelbs), Advances in Probability and Related Topics Vol. 4 (Edited by P. Ney), 267-517, Marcel Dekker, New York (1978). 